\title{
Sleep-disordered breathing and cardiovascular disease: an epiphenomenon or a causal relationship?
}

\author{
Takatoshi Kasai ${ }^{1,2}$
}

Published online: 2 August 2017

(C) Japanese Society of Sleep Research 2017

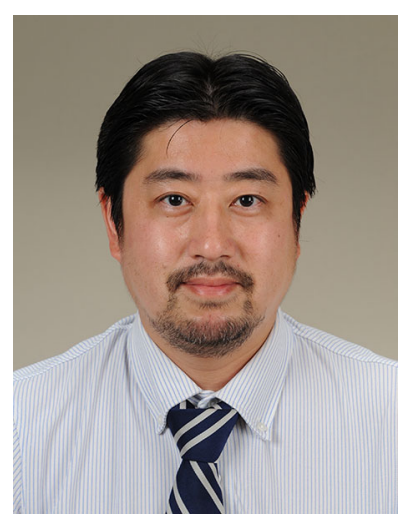

Sleep-disordered breathing (SDB) has become a worldwide public health concern. In Japan, an estimated 400,000 patients with SDB use continuous positive airway pressure (CPAP) devices, and the number of CPAP users is increasing annually [1].

In the late 1980s, observational studies in North America reported that SDB is not just a disorder that causes excessive daytime sleepiness but is also associated with increased mortality. Then, it was suggested that the high mortality rate in patients with SDB can be explained by the presence of comorbid cardiovascular disease (CVD). In a cross-sectional analysis of the Sleep Heart Health Study (SHHS), SDB showed a significant association with self-

Takatoshi Kasai

kasai-t@mx6.nisiq.net

1 Department of Cardiovascular Medicine, Juntendo University School of Medicine, Tokyo, Japan

2 Cardiovascular Respiratory Sleep Medicine, Juntendo University Graduate School of Medicine, 2-1-1 Hongo, Bunkyo-ku, Tokyo 113-8421, Japan reported CVD, independent from other confounding factors, suggesting that SDB might not be just an epiphenomenon but a cause of CVD. Indeed, a number of studies showed that SDB has adverse cardiovascular effects, which may link SDB with the development of CVD. Conversely, small-scale and/or short-term randomized controlled trials (RCTs) suggested that SDB treatment with positive airway pressure (PAP) had beneficial effects on surrogate cardiovascular endpoints such as blood pressure, left ventricular function, and cardiovascular biomarkers.

In the 2000s, longitudinal analyses of epidemiological and observational studies provided confirmatory data regarding SDB-CVD causality. For instance, in the SHHS and in the Wisconsin Sleep Cohort, risks for both all-cause and cardiovascular mortality in subjects with severe SDB were significantly greater than in those without SDB. In several observational studies in which patients with CVD, including coronary artery disease and heart failure (HF), were enrolled, SDB was highly prevalent and the presence of SDB was associated with increased risk of mortality and/or cardiovascular events. More recently, for further confirmation of SDB-CVD causality, whether the alleviation of SDB by PAP can improve cardiovascular outcomes has been investigated in several RCTs enrolling patients with or without CVD or both. However, none of these RCTs have shown a beneficial effect of PAP treatment on cardiovascular outcomes, in terms of primary composite endpoints. In addition, a current meta-analysis including 10 RCTs that investigated the effects of PAP in patients with and without CVD did not find a significant association between SDB treatment with PAP and better cardiovascular outcomes [2].

Considering these negative results, the following question is raised: "Does this indicate a lack of SDB-CVD causality?" My answer is "No". In fact, several 
issues/possibilities have already been raised to account for such unexpected results and to explain why these RCTs failed to show cardiovascular benefits. For example, sleepy patients were excluded, there was variable compliance with treatment devices, and crossover between control and intervention groups was too large. Further RCTs investigating the effects of SDB treatment with PAP on cardiovascular outcomes are currently underway. These include the Impact of Sleep Apnea Syndrome in the Evolution of Acute Coronary Syndrome (ISAACC) trial, which is investigating the effects of CPAP on cardiovascular outcomes in patients with SDB and acute coronary syndrome; the multi-center, randomized study to assess the Effect of Adaptive Servo Ventilation on Survival and Frequency of Hospital Admissions in Patients with Heart Failure and Sleep Apnea (ADVENT-HF) trial, which is investigating the effects of treating SDB with adaptive servo-ventilation on cardiovascular outcomes in patients with HF; and the Sleep-Disordered Breathing in Transient Ischemic Attack/ Ischemic Stroke and Continuous Positive Airway Pressure Treatment Efficacy (SAS-CARE) trial, which is investigating the effects of CPAP on cerebrovascular outcomes in patients who have experienced an acute cerebrovascular event. I believe that SDB-CVD causality will be validated by these trials. However, it will take at least several years to see results in these RCTs.

In the meantime, original research other than long-term RCTs regarding the effects of PAP on cardiovascular outcomes in patients with SDB should be pursued to provide novel insights and generate further hypotheses in the field of CVD and SDB. In addition, RCTs investigating the effects of SDB treatment with PAP on cardiovascular outcomes from Asian countries are still needed to confirm applicability of data from Western countries. Sleep and Biological Rhythms [3] is a right place for such research papers. In the upcoming decade, many such research papers, especially from Asian countries, will hopefully be published in Sleep and Biological Rhythms and will be read and discussed worldwide.

\section{Compliance with ethical standards}

Funding None.

Conflict of interest T Kasai is affiliated with a department endowed by Philips Respironics, ResMed, Teijin Home Healthcare, and Fukuda Denshi.

\section{References}

1. Chin K. Interactions among sleep disordered breathing, obesity, and sleep duration. Sleep Biol Rhythms. 2017;15:87-8.

2. Yu J, Zhou Z, McEvoy RD, Anderson CS, Rodgers A, Perkovic V, et al. Association of positive airway pressure with cardiovascular events and death in adults with sleep apnea: a systematic review and meta-analysis. JAMA. 2017;318:156-66.

3. Uchiyama M. Preface. Sleep Biol Rhythms. 2017;15:1-2. 